\title{
El análisis del coste del ciclo de vida como herramienta para la evaluación económica de la edificación sostenible. Estado de la cuestión
}

\author{
Life cycle cost analysis as an economic evaluation tool for sustanaible \\ building. Sate of the art \\ F. García-Erviti $^{(*)}$, J. Armengot-Paradinas ${ }^{(*)}$, G. Ramírez-Pacheco ${ }^{(*)}$
}

\section{RESUMEN}

El presente trabajo expone el estado actual de la cuestión en referencia al análisis del coste del ciclo de vida (CCV) aplicado a la edificación. Las diferentes metodologías desarrolladas en torno a este concepto constituye una herramienta destinada a la evaluación de las distintas opciones de proyecto desde una perspectiva de eficiencia económica medioambientalmente sostenible. Basado en la obtención del valor actual de los costes y beneficios futuros asociados a las decisiones del proceso de edificación, el análisis CCV representa un cambio de paradigma: opone una visión a largo plazo frente a la perspectiva tradicional que aspira a la obtención de una rentabilidad inmediata con una mínima inversión inicial, ignorando sus efectos económicos y medioambientales futuros. El objetivo del artículo es la revisión de la metodología CCV y su implantación en un contexto global, analizando su proceso de desarrollo y los criterios de discusión de resultados. Se concluye con un estudio crítico de las posibilidades de adaptación del método a la estructura del sector inmobiliario español, proponiendo su integración en un sistema de contratación pública sostenible.

Palabras clave: Coste del ciclo de vida; evaluación; viabilidad económica; contratación pública sostenible.

\section{ABSTRACT}

Life-Cycle Costing (LCC) applied to construction building is a tool used for the evaluation of different project options from the perspective of economic efficiency environmentally sustainable. This method is based on obtaining the present value of future costs and benefits linked with decisions of the building process. The LCC analysis represents a paradigm change. It opposes a long-term vision with the traditional approach of obtaining immediate returns with a minimal initial investment, ignoring their economic and environmental future effects. This paper reviews the implementation of the LCC methodology in a global context, analyzing the development process and criteria for the evaluation of results. It concludes with a critical study of the possibilities of adapting the method to the structure of the Spanish property market, suggesting their integration into a sustainable public procurement system.

Keywords: Life-cycle costing; evaluation; economic feasibility; sustainable public procurement.

(*) E.T.S. Arquitectura - Universidad Politécnica de Madrid (España).

Persona de contacto/Corresponding author: federico.garcia@upm.es (F. García-Erviti)

Cómo citar este artículo/Citation: García-Erviti, F., Armengot-Paradinas, J., Ramírez-Pacheco, G. (2015). El análisis del coste del ciclo de vida como herramienta para la evaluación económica de la edificación sostenible. Estado de la cuestión. Informes de la Construcción, 67(537): e056, doi: http://dx.doi.org/10.3989/ic.12.119.

Licencia / License: Salvo indicación contraria, todos los contenidos de la edición electrónica de Informes de la Construcción se distribuyen bajo una licencia de uso y distribución Creative Commons Reconocimiento no Comercial 3.o. España (cc-by-nc). 


\section{INTRODUCCIÓN}

En el papel que hoy desempeña en España el sector de la edificación, y el de la construcción en general, el paradigma de la eficiencia en la gestión de unos recursos cada vez más escasos y del rigor extremo en el control del endeudamiento público se abre paso después de una era caracterizada por la cultura del despilfarro, en la creencia de que los recursos disponibles eran inagotables. La teoría de las decisiones sobre el uso de recursos limitados, que se sitúa en el núcleo de la ciencia económica, entronca así con el concepto de construcción sostenible. Esta idea se ha traducido en la aplicación de nuevas herramientas para la evaluación de la aportación de las infraestructuras, edificios y procesos constructivos al desarrollo sostenible utilizando resultados cuantitativos concretos, como se ha puesto de manifiesto en estas páginas (1). En esta tarea, el método de análisis del coste del ciclo de vida, CCV (LCC en la sigla inglesa de Life-Cycle Costing ${ }^{1}$ ), se convierte en una herramienta fundamental en el proceso de toma de decisiones para la evaluación de proyectos económica y medioambientalmente viables, en un análisis realizado desde la concepción hasta el término de la vida útil de la edificación o infraestructura proyectada.

\section{EL ANÁLISIS ECONÓMICO Y EL CICLO DE VIDA EN LA EVALUACIÓN DE LA SOSTENIBILIDAD DE LOS EDIFICIOS}

Apoyándose en la definición de Bruntland (1987), «desarrollo sostenible es el que satisface las necesidades presentes sin comprometer la capacidad de generaciones futuras de satisfacer sus propias necesidades» (2), la literatura sobre la materia se ha puesto de acuerdo históricamente en que la sostenibilidad depende de tres factores: medio ambiente, economía y sociedad: la concurrencia de estos tres elementos permitirá afirmar de forma rotunda que un bien -en nuestro caso, un edificio, un sistema constructivo o un elemento de un edificio- es sostenible.

Por otra parte, el estudio de la sostenibilidad se afronta desde la consideración de los elementos durante su ciclo de vida de la cuna a la tumba, lo que hace posible comparar diversas opciones. Para la evaluación del ciclo de vida se han desarrollado diferentes metodologías, dependiendo de si se cuantifica el coste ambiental del proceso de producción que se conoce como Análisis del Ciclo de Vida (ACV) ${ }^{2}$, o el coste económico, cuyo análisis se desarrolla a través del Coste del Ciclo de Vida (CCV).

El CCV incorpora a su vez el desempeño medioambiental del proceso, y está directamente relacionado con el ACV. Según la Norma UNE-EN ISO 1404O: Gestión ambiental. Análisis de ciclo de vida. Principios y marco de referencia, el ACV consiste en un análisis de los impactos ambientales potenciales que, tanto en la utilización de recursos como en las consecuencias de las emisiones, se producen a lo largo del ciclo de vida de un producto o sistema de producción. Este análisis requiere la realización de un inventario de las aportaciones de energía y materiales durante todo el ciclo de vida, así como de las transferencias al medio ambiente, de forma que la interpretación de los resultados permita una evaluación sistemática de los impactos. Por el contrario, el CCV se aleja del concepto de interpretación del inventario de aportaciones y pérdidas de los distintos elementos del sistema, dirigiendo su interés hacia un análisis coste-beneficio en el contexto de la eficiencia energética y del rendimiento medioambiental del proyecto a lo largo de la vida del activo.

Por tanto, el CCV no es una herramienta de medición exclusivamente ambiental, aunque incorpore el concepto de «ciclo de vida» (3). Conviene recordar que la sostenibilidad es algo más que ecología y, por ello, el CCV puede considerarse un instrumento útil para la evaluación de la sostenibilidad (4), frente a la posición contraria mantenida en ocasiones (5). No en vano, desde iniciativas como SMART SPP (6), se están desarrollando aplicaciones para la medición conjunta de impacto ambiental y económico, como la herramienta CCV$\mathrm{CO} 2$, orientada a la baremación de propuestas en licitaciones públicas. Por la subjetividad que implican los resultados en términos de emisiones, el propio manual de la herramienta deja en manos del evaluador la consideración de los mismos, como un argumento más para ponderar las cifras económicas.

En esta línea, el reciente enfoque normativo sobre la evaluación de edificios desde el punto de vista de la sostenibilidad en la edificación, desarrollado en la norma europea EN 15643, plantea un análisis integral del ciclo de vida de los edificios desde el punto de vista del desempeño medioambiental, social y económico. Estos elementos constituyen los denominados «tres pilares» del desarrollo sostenible, concepto basado en los principios de la Agenda 21 de Rio y en el Plan de Aplicación de las decisiones de la Cumbre Mundial sobre desarrollo sostenible de Johannesburgo 2002. Este triple enfoque, que combina ACV económico (en el que se integra el análisis CCV que aquí se trata), ACV ambiental y ACV social, inspira el proyecto CILECCTA (7), cofinanciado por la Comisión Europea bajo el $7^{\circ}$ Programa Marco, al que también nos referiremos después.

\section{EL ANÁLISIS CCV: UNA VISIÓN GLOBAL}

El análisis del coste del ciclo de vida en el sector de la edificación tiene su inicio en las políticas institucionales que en los años setenta del siglo pasado dirigían los gobiernos británico y norteamericano hacia el ahorro de energía en los edificios de sus respectivas administraciones públicas. En 1977, el Departamento de Industria del gobierno británico publicó una guía práctica sobre la aplicación del coste del ciclo de vida en la gestión de su patrimonio inmobiliario (8). Un año más tarde, el National Bureau of Standards estadounidense editó una guía para la selección de proyectos de ahorro energético en edificios públicos mediante la aplicación de la misma técnica de evaluación de costes (9). En 1987, el National Institute of Standards and Technology (NIST) norteamericano publicó el documento titulado Life-Cycle Costing for the Federal Management Program, actualizado en 1995 (10), que anualmente se complementa mediante la publicación de los índices de precios de la energía y tasas de descuento para la realización

\footnotetext{
${ }^{1}$ En este trabajo se adoptará la sigla CCV, traducción de la correspondiente en lengua inglesa LCC, la más utilizada en la UE y en la mayor parte del resto del mundo. Otras acepciones sinónimas, como LCCA (Life-Cycle Cost Analysis), utilizada por el National Institute of Building Sciences norteamericano, o WLCC (Whole Life-Cycle Costing), con origen en Gran Bretaña, tienen una aplicación más limitada.

${ }^{2}$ LCA (Life-Cycle Assessment) en su traducción a la lengua inglesa.
} 
del análisis del coste del ciclo de vida (11). Paralelamente, el análisis CCV se ha ido aplicando a la mayor parte de los procesos de licitación, adjudicación, construcción y gestión de edificios de las distintas administraciones federales de los EEUU, así como en las instituciones gubernamentales de otros países, como Canadá, Australia o Nueva Zelanda (12) (13).

En cuanto a la acción política conjunta de los países miembros de la UE en relación con el CCV, en abril de 2001 se inició el proyecto EuroLifeForm destinado al desarrollo de un «Método probabilístico para la predicción del coste del ciclo de vida y las prestaciones de los edificios y las infraestructuras civiles» (14), que se concretaba en el desarrollo de un procedimiento compuesto por tres elementos distintos: un modelo probabilístico de costes del ciclo de vida, un modelo del deterioro y una aplicación de soporte de decisiones. Además, incorporaba un análisis elemental de impacto ambiental a desarrollar durante todo el proceso de diseño.

En 2003 se publicó el Informe Final del Grupo de Trabajo TG4 de la Comisión Europea relativo a los costes del ciclo de vida en la construcción (15), en el que estaba representado el Ministerio de Fomento español, figurando la empresa Dragados Concesiones como experto independiente. Entre las recomendaciones del informe figura la adopción de una metodología común para la aplicación del CCV en la Unión Europea, así como la impulsión de la creación de bases de datos de referencia y, en especial, la incorporación del CCV al sistema de contratación pública (Figura 1).

Como consecuencia de la actividad del TG4, en el proceso de búsqueda de un procedimiento unificado aplicable a los países miembros de la UE, en 2007 se publicó un informe realizado por la consultora Davis Langdon por encargo de la Comisión Europea (16), destinado a la búsqueda de las posibilidades de desarrollo de una metodología común para la aplicación del análisis CCV en los países miembros. En cuanto a la implantación del sistema en cada país, el informe de Davis Langdon pone de manifiesto una escasa difusión en el ámbito de la promoción privada y una notable dispersión en su aplicación en las políticas públicas de las distintas instituciones estatales, federales o locales, revelando la descoor-

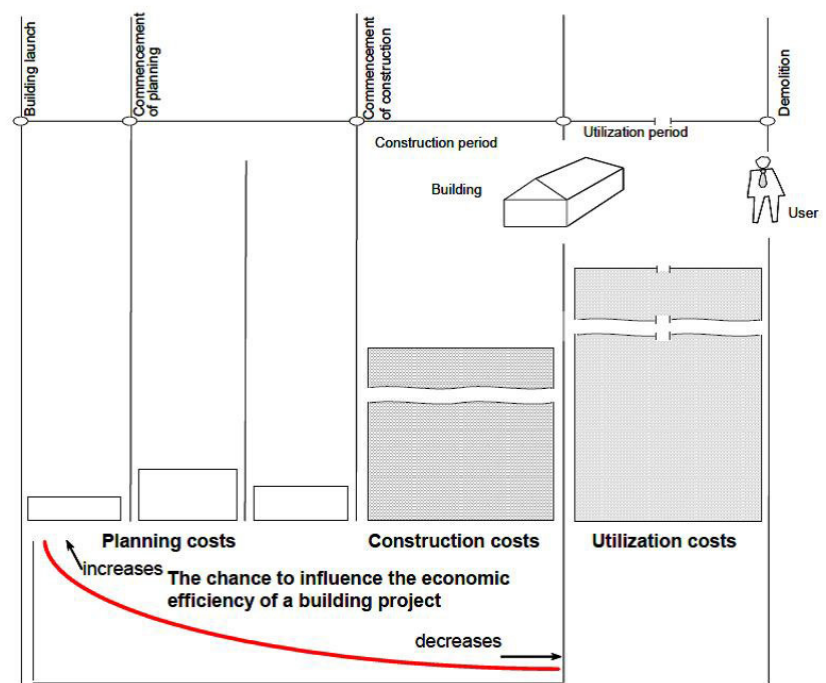

Figura 1. El coste del ciclo de vida en la construcción. Fuente: Task Group 4. Life-cycle cost in construction. Final Report. European Commission. dinación existente, incluso dentro de los países en los que el desarrollo de la metodología es más intenso. El informe constata que en el ámbito institucional, la única normativa publicada a nivel estatal sobre el coste del ciclo de vida en la edificación es la Noruega (17).

Un año después de la emisión del citado informe de Davis Langdon se publicó la primera edición de la Norma ISO 15686-5: Edificaciones y bienes inmuebles construidos Planificación de la vida útil. Parte 5: Coste del ciclo de vida (18). Planteando entre sus objetivos la mejora de los procesos de toma de decisiones y de evaluación de resultados en las distintas etapas de desarrollo del proyecto, la ISO 15686-5 define el coste del ciclo de vida como una metodología para predecir los costes de los bienes inmuebles construidos, de acuerdo con los requerimientos del promotor, entendiendo por tal tanto al empresario que desarrolla una promoción o gestiona un patrimonio edificado de naturaleza privada como, en especial, a las instituciones responsables de la gestión patrimonial de las administraciones públicas.

Esta norma relaciona los costes incluidos en el análisis, diferenciando los correspondientes al ciclo de vida que podría denominarse «elemental» (LCC), de los propios del ciclo de vida integral (Whole-Life Cost, WLC). Entre los primeros se incluyen los de la construcción, funcionamiento, mantenimiento y demolición del edificio; por su parte, el WLC incorpora desde los costes e inversiones externos al proceso constructivo, aunque imprescindibles para el funcionamiento del edificio, hasta los financieros y fiscales; incluye también los ingresos generados por el inmueble a lo largo de la vida útil, entre los que incorpora el valor residual del suelo al final de la misma, una vez desaparecido el edificio (Figura 2). La norma ISO, por último, propone pautas para la aplicación de técnicas estadísticas en la gestión de las variables de riesgo e incertidumbre para la estimación de situaciones futuras. Como suplemento a la norma ISO, el BCIS (Building Cost Information Service) británico publicó en el mismo año 2008 un método normalizado para la aplicación del análisis CCV en la contratación de obras de construcción (19), que no tiene el carácter de norma pero que es una referencia habitual en el sector en el Reino Unido.

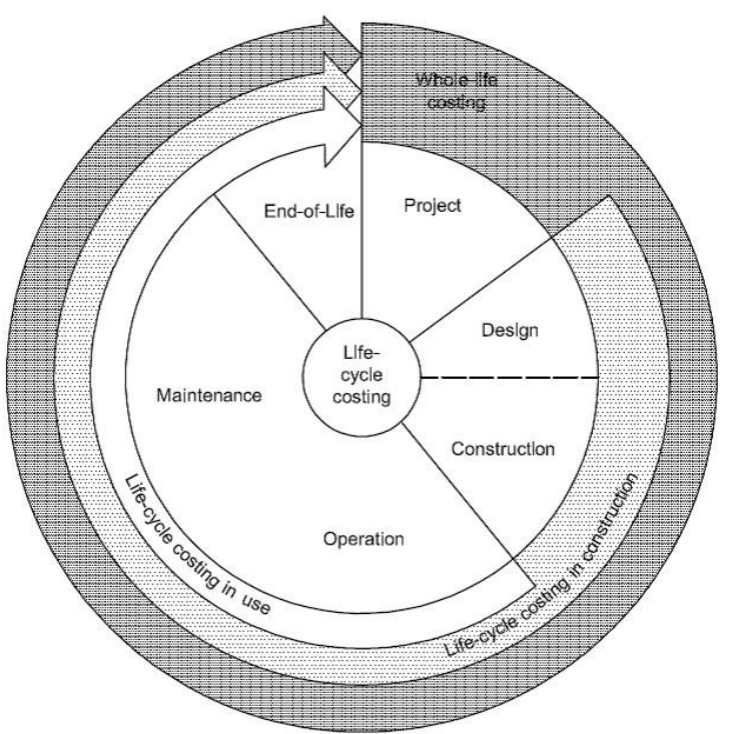

Figura 2. Análisis de las diferentes etapas del ciclo de vida. Fuente: ISO 15686-5. 
Cofinanciado por la Comisión Europea en el $6^{\circ}$ Programa Marco (2006-2010), el proyecto InPro Building (20) desarrolló una metodología de «construcción virtual» durante las etapas preliminares del proceso de proyecto. Este procedimiento se basa en la aplicación de sistemas BIM (Building Information Modeling) consistentes en un análisis tridimensional que incorpora información de todos los elementos y de la repercusiones de cada decisión de proyecto en cada uno de ellos, incluidas las económicas y financieras durante las etapas futuras de la vida del edificio proyectado.

En 2010, la consultora Davis Langdon emitió un nuevo informe para la Comisión Europea sobre el desarrollo de una campaña promocional del coste del ciclo de vida en la construcción (21). En este documento, que desarrolla un estudio aplicado a once países europeos entre los que no se encuentra España, se constata que todos ellos tienden a confluir en una estructura unificada de costes adaptada a la citada Norma ISO 15686-5; la información sobre los datos de costes y prestaciones se obtiene, sin embargo, a partir de una multiplicidad de fuentes, predominantemente privadas, con resultados muy dispares; la propuesta de metodología unificada se aplicó a 15 proyectos, adaptándose sin dificultad a las prácticas de aplicación del análisis CCV en cada país analizado. El informe afirma que la organización de una estructura unificada de costes parece inviable a nivel $\mathrm{CE}$, debido tanto a las diferencias que presentan los distintos sistemas y técnicas constructivos utilizados como a la diversidad de normas locales; no obstante, la confluencia de varios países en las propuestas de la Parte 5 de la ISO 15686 parece aconsejar una adopción de estos criterios con carácter generalizado en el futuro.

La última iniciativa institucional directamente vinculada al análisis CCV procede de la Directiva 2010/31/UE, uno de cuyos objetivos es establecer un marco metodológico comparativo para la determinación por los estados miembros de los niveles óptimos de rentabilidad de los requisitos de eficiencia energética de los edificios y sus elementos. En desarrollo de esta Directiva se ha publicado el Reglamento Delegado $\mathrm{n}^{0}$ 244/2012 y las Directrices que lo acompañan, documentos en los que se establece un método de análisis del coste global de los costes e ingresos a realizar durante el período de vida útil de los edificios o elementos para el cumplimiento de los citados requisitos de eficiencia energética. Dicho método, consistente en la obtención del valor actual neto de los costes e ingresos de cada edificio tipo para compararlo con el que es objeto de análisis, "permite el uso de un período de cálculo uniforme (en que los equipos de vida más larga se tienen en cuenta a través de su valor residual) y que puede hacer uso del coste del ciclo de vida (CCV), que se basa también en cálculos de valor actual neto» (Directrices, 6).

En la idea de dar un paso más en la medición global de la sostenibilidad surge una metodología común europea para el desarrollo del concepto de Análisis de la Sostenibilidad en el Ciclo de Vida -ASCV integral- (22), que combina CCV, ACV ambiental, y ACV social. Se sigue así una estrategia basada en los principios de la Agenda 21 de Rio y desarrollada después por la Cumbre Mundial 2005, cuyo documento final manifiesta que «el desarrollo sostenible en sus aspectos económico, social y medioambiental constituye un elemento clave en las actividades de las Naciones Unidas». Este triple enfoque es el que inspira la norma europea EN 15643 (23), que en 2012 está en proceso de adaptación a la normativa española (24), en cuyo marco se publica la EN 15643-4 Asessment of Buildings
- Part 4: Framework for the asessment of economic performance, que regula las prestaciones económicas de los edificios en relación con sus características técnicas y funcionales, asumiendo que la evaluación económica es parte integrante de la evaluación de la sostenibilidad de la edificación.

Entre las iniciativas europeas encaminadas a la mejora de la eficiencia energética en la edificación, se impulsa el proyecto Factor 4 entre 2005 y 2008 por la Agencia de la Competitividad y la Innovación de la Comisión Europea. Enfocado hacia la rehabilitación de viviendas sociales, este proyecto se proponía, aplicando medidas de mejora energética y mediante el uso de energías renovables, reducir a la cuarta parte las emisiones de gases de efecto invernadero antes de 2050. Su objetivo era apoyar a los propietarios en los procesos de optimización de los programas de rehabilitación, aplicando para ello el método de análisis del coste del ciclo de vida de los recursos energéticos, que permite enfatizar las ventajas de las medidas de mejora en la eficiencia energética, tanto en lo que se refiere a los períodos de retorno de las inversiones para los propietarios, como a la reducción de costes de funcionamiento para los inquilinos.

Siguiendo esta línea de trabajo nace en 2010 el ya citado proyecto CILECCTA, al que también nos hemos referido antes, cofinanciado por la Comisión Europea bajo el $7^{\circ}$ Programa Marco, cuya finalización está prevista en 2013. Este proyecto, en el que participan la consultora española APIA XXI s.a. (25) y Acciona Infraestructuras S.A., tiene por objeto el desarrollo de herramientas informáticas que permitan la evaluación y gestión de proyectos de construcción sostenibles que incorporen estrategias flexibles para sostenibilidad a largo plazo sujetas a cierto grado de incertidumbre (26). El proyecto introduce el concepto de evaluación y cuantificación de incertidumbres y la integración de los análisis CCV y ACV, en un informe denominado ecoportfolio (Figura 3).

El programa permite proponer ciclos de vida alternativos, en fase de proyecto, y diferentes escenarios para su implantación durante la vida útil. Los métodos tradicionales de CCV y ACV parten de la base de que es posible tener datos fiables del futuro. Pero la incertidumbre obliga a pasar de modelos deterministas a modelos probabilísticos, que recojan la probabilidad de cambio en factores como coste y durabilidad. El modelo determinista exige datos concretos para todas las variables, pero lo más realista es manejar rangos de valores, lo que conduce a horquillas o bandas variables de resultados (modelo probabilístico). Por otro lado, el modelo probabilístico puede ligarse

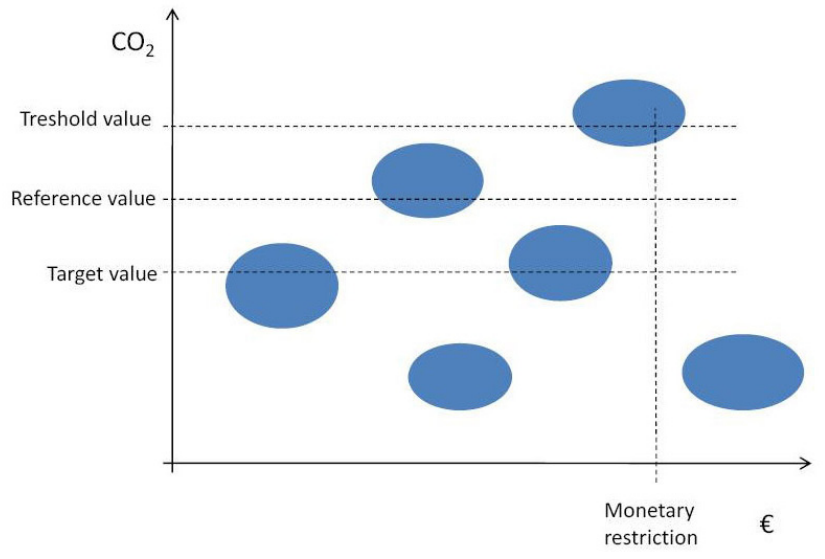

Figura 3. Ejemplo de ecoportfolio. Fuente: CILECCTA. 
con un modelo de Ciclos de Vida Alternativos. Como hipótesis de partida, la flexibilidad aumenta el valor de un activo al reducir el impacto negativo de la incertidumbre, y a mayor incertidumbre, mayor valor aporta la flexibilidad del edificio.

\section{EL ANÁLISIS CCV: METODOLOGÍA}

En síntesis, los elementos clave del análisis CCV son cuatro: la determinación de los flujos financieros de cobros y pagos costes y beneficios, en términos de evaluación de proyectos-, el horizonte temporal del proyecto, las tasas de descuento o actualización financiera aplicables y el método de análisis de resultados. El objetivo básico es la obtención del valor actual neto de todos los flujos financieros del proceso de edificación. Un análisis de coste de vida global incorpora, por lo tanto, todos los costes e inversiones producidos desde la adquisición o disposición del terreno: los estudios de viabilidad de su desarrollo futuro; la gestión de la obtención de toda clase de autorizaciones administrativas necesarias; los honorarios técnicos de proyecto, dirección de obra, gestión integrada y otras intervenciones profesionales; los costes fiscales y financieros generados por la construcción y los costes intrínsecos de esta; los costes de mantenimiento, funcionamiento, rehabilitación y sustitución de elementos y sistemas durante la vida útil del edificio y, finalmente, deben incorporarse los costes e inversiones necesarios para la demolición, gestión de residuos y, en su caso, reutilización de los elementos recuperables. En la corriente de flujos opuestos se contabilizan los ingresos generados durante la vida útil del inmueble, generalmente representados por las rentas, arrendaticias o de otra índole, que realmente producirá o será susceptible de producir en el futuro. En el caso de los edificios promovidos por las Administraciones públicas o destinados al uso propio del promotor o de los sucesivos adquirentes, estos ingresos se identificarán con las rentas que habrían tenido que satisfacer si se hubiera tenido que arrendar un edificio análogo. En los análisis CCV destinados a la optimización de la eficiencia energética se prescinde de estos rendimientos de explotación, quedando restringidos los ingresos a los generados por la energía exportada, según la propuesta de la Directiva 2010/31/UE. Por último, debe estimarse el valor residual actual de aquellos elementos y sistemas que sean recuperables o reutilizables, que vendrá determinado por la depreciación del coste de inversión inicial o del coste de sustitución, durante el ciclo de vida útil de dichos elementos o sistemas (27).

Para definir el alcance de los costes y beneficios de una determinada decisión, la Directiva 2010/31/UE plantea una doble visión que comprenda, además de una perspectiva de carácter financiero, una perspectiva macroeconómica. La perspectiva financiera considera únicamente los costes y beneficios inmediatos de la decisión de inversión, pero existen otra serie de conceptos económicos indirectos que afectan a actores del mercado distintos del inversor y que obligan a incorporar una perspectiva macroeconómica en el análisis. El objetivo del análisis macroeconómico es englobar las inversiones en una perspectiva más amplia del bien público, integrando las ventajas directas e indirectas para la sociedad, planteamiento que entronca con el triple enfoque del desempeño medioambiental, social y económico desarrollado por la normativa europea citada.

Desde una perspectiva macroeconómica deben incorporarse determinadas externalidades de naturaleza contingente, entre los que se incluyen los beneficios derivados de la repercusión (rentabilidad o perjuicio) social de determinadas deci- siones de promoción o inversión, en especial las derivadas de políticas públicas. Se contabilizan también, entre estos conceptos relativamente intangibles, los costes marginales sociales producidos por las externalidades negativas (por ejemplo, el impacto medioambiental de decisiones constructivas energéticamente ineficientes), además de los costes de oportunidad de la renuncia a soluciones alternativas.

La determinación del horizonte temporal constituye una de las decisiones más complejas del análisis CCV. Se trata de establecer la duración o vida económica del proyecto y de sus distintos elementos, cuestión fundamental en un método basado en el análisis de los efectos del transcurso del tiempo en el valor de los distintos flujos de costes y beneficios. La Norma ISO 15686-5 se limita a señalar que la vida útil del proyecto es un dato que forma parte del programa de necesidades del promotor del edificio, diferenciándolo del concepto de «período de análisis». Las Directrices Complementarias al Reglamento Delegado ${ }^{\circ}$ 244/2012 de la repetida Directiva 2010/31/UE establecen un periodo de cálculo de 30 años para los edificios residenciales-públicos y 20 años para las edificaciones no residenciales-comerciales.

Pero el problema no se limita a determinar la vida total del proyecto, porque también es preciso establecer la duración de cada uno de los elementos, equipos o sistemas de cara a su sustitución y renovación, cuestión relevante cuando se trata de comparar proyectos alternativos que plantean distintas soluciones constructivas y tecnológicas. La Norma ISO 15686 Buildings and Constructed Assets: Service Life Planning; Part 1: Service Life Prediction Procedures propone un método factorial de estimación de la duración de los distintos componentes de un edificio en función de su calidad, nivel de diseño y ejecución, exposición medioambiental y condiciones de uso y mantenimiento, que es una simplificación del procedimiento de cálculo desarrollado en la norma Principal Guide for Service Life Planning of Building publicada por el Architecture Institute of Japan -AIJ- (28). Además, varios estándares británicos establecen criterios de durabilidad de los distintos componentes de los edificios, en especial la BS7543:2003 Guide to Durability of Buildings and Building Elements, Products and Components, una de las normas pioneras sobre la materia junto a la japonesa y la Canadian Standard S478-1994 Guideline on Durability in Buildings. Por otra parte, la mayor parte de las entidades hipotecarias globales manejan datos sobre vidas medias estimadas de los distintos componentes de los edificios, y en Estados Unidos muchos gobiernos federales y Universidades desarrollan modelos ad-hoc para evaluar el inventario de los bienes inmuebles de sus respectivas instituciones (29) (30) (31). En todo caso, no debe olvidarse que el análisis LCC no solo se aplica a la construcción de edificios completos, sino que también se utiliza en el desarrollo de elementos y sistemas constructivos, con múltiples ejemplos en la literatura científica (32) (33) (34) (35). Por ello, algunos fabricantes europeos de determinados elementos de tecnología avanzada han desarrollado sus propios análisis LCC, para permitir una elección económicamente eficiente y medioambientalmente responsable de sus productos.

El indicador de rentabilidad a emplear dependerá del alcance del análisis: si únicamente se pretende obtener el período de tiempo necesario para recuperar o amortizar las inversiones iniciales se calculará el plazo de recuperación de la inversión o pay-back. Este método de análisis estático no tiene en cuenta la dimensión temporal de los flujos financieros del proceso 
de edificación a lo largo del ciclo de vida. El procedimiento dinámico que permite comparar flujos financieros (beneficios y costes, ingresos y gastos, cobros y pagos) que tienen lugar en diferentes momentos temporales, convirtiendo los flujos futuros en valores actuales, se basa en la utilización de la tasa de descuento. El descuento de flujos representa la preferencia por recibir bienes y servicios con la mayor anticipación posible, difiriendo los costes al futuro, por lo que, en la evaluación de proyectos públicos, el tipo de descuento aplicable a los costes y beneficios futuros recibe el nombre de «tasa social de preferencia temporal». La tasa de descuento se identifica con la rentabilidad libre de riesgo (que generalmente se asimila a la de los títulos de deuda pública), incrementada en una prima que remunere el riesgo diferencial característico de cada proyecto.

Adoptadas las hipótesis de flujos, desarrollo temporal y tasa de descuento, la discusión de resultados requiere, como todos los desarrollos a largo plazo, una estimación de incertidumbre y riesgos asociados, junto con un análisis de sensibilidad que permita discernir el impacto que se produce en los resultados como consecuencia de las hipótesis adoptadas para las variables más sensibles, en especial en lo relativo a la determinación de la tasa de descuento. En cuanto al análisis de resultados propiamente dicho, no debe olvidarse que el CCV es un método de análisis comparativo entre distintas opciones posibles. Por ello, son múltiples los parámetros a utilizar para la selección de la solución óptima; el instrumento de medida típicamente utilizado en la evaluación de proyectos es el valor actual neto (VAN, o NPV en su sigla en inglés) de los flujos de caja futuros del proyecto. Pero alternativamente pueden utilizarse ratios de beneficio obtenidas sobre la inversión total realizada durante la vida del proyecto, análisis de reducción de costes anuales, sistemas de pay-back o plazos de recuperación de la inversión dinámicos, tasas de rentabilidad del proyecto o tasas interna de retorno en relación con el coste de capital invertido (TIR en español) o, finalmente, los costes anuales equivalentes por proyecto, entre otros. Existen estudios comparativos sobre las ventajas e inconvenientes de cada método de análisis y la idoneidad de cada uno de ellos en relación con la finalidad de la valoración (36).

Son múltiples las herramientas informáticas disponibles para la realización de análisis CCV, y en Davis Langdon 2007 hay una revisión de las más significativas (37). La más reciente es la que propone el también citado proyecto europeo SMART, destinado a la implantación del análisis CCV en los sistemas de contratación pública sostenible, en el que participa el Ayuntamiento de Barcelona.

\section{EL ANÁLISIS CCV EN ESPAÑA: ESTADO ACTUAL}

El repetido informe de 2007 de Davis Langdon constata, en el contexto de una revisión de la aplicación de la metodología CCV en los países miembros de la UE, que la legislación española de contratos para las administraciones públicas (en referencia al Texto Refundido aprobado por Real Decreto Legislativo 2/2000, que precedía a la Ley 30/2007, de 30 de octubre, de Contratos del Sector Público actualmente vigente) constituye una combinación de aspectos legales, técnicos y contractuales completamente favorable para la aplicación de la metodología CCV a los contratos de obra pública. Reseña este informe, además, que alguna empresa privada española del sector de la construcción aplica procedimientos propios con carácter interno, advirtiendo que no existe el soporte institucional necesario.

Siendo cierta la apreciación anterior para la aplicación del análisis CCV a los proyectos españoles de infraestructuras y obra civil, el informe de Davis Langdon no tiene en cuenta, sin embargo, uno de los rasgos característicos del sector de la promoción inmobiliaria en España, determinante del estado actual de la aplicación de los análisis CCV en el ámbito de la edificación en nuestro país, en el que la vivienda en alquiler no supera el $15 \%$ del parque residencial ${ }^{3}$, frente a la media europea del $36 \%$. Esta realidad es trasladable a algunos usos terciarios, lo que hace minoritarias las inversiones de tipo patrimonial en el mercado inmobiliario local, en las que el promotor soporta durante toda la vida útil del inmueble los riesgos y costes derivados de un erróneo planteamiento inicial del proyecto de inversión.

Por otra parte, la Ley de Contratos del Sector Público española tampoco ha incorporado el análisis CCV como elemento de juicio en el criterio de valoración de las ofertas que forma parte del proceso de selección del adjudicatario. No se han seguido así hasta ahora las recomendaciones que se han realizado por los distintos organismos y grupos de trabajo de la Unión Europea en este sentido. Como consecuencia de los trabajos del antes citado Grupo de Trabajo TG4 "Costes del Ciclo de Vida en la Construcción y sus recomendaciones de aplicación», en la Comunicación de 2004 de la Comisión Europea al Consejo titulada «Hacia una estrategia temática sobre el medio ambiente urbano», se proponía por primera vez la aplicación coordinada del método CCV en los países miembros: «La Comisión desarrollará una metodología común para evaluar la sostenibilidad global de los edificios y del entorno construido, que incluirá indicadores de costes durante el ciclo de vida útil. Ésta se aplicará también a los proyectos de edificios nuevos y a las renovaciones importantes. Se alentará a todos los Estados miembros a adoptar esta metodología y a utilizarla como soporte de las mejores prácticas».

El documento Buying Green!. Manual de contratación pública medioambiental, publicado por la Comisión Europea en el mismo año 2004 (38), concluye afirmando que «la adopción de una metodología del coste del ciclo de vida revela los verdaderos costes de un contrato", así como que "el uso de este método en la preparación de los criterios de adjudicación mejorará tanto las prestaciones medioambientales como los aspectos financieros del proceso de contratación». Para ello se proponen diversas estrategias de aplicación del método en los sistemas de contratación pública. Finalmente, en 2008, una Comunicación de la Comisión Europea titulada «Contratación pública para mejorar el medioambiente» (39) señala a la construcción como el primer sector prioritario para la aplicación de políticas de esta naturaleza, implantando la estrategia GPP (Green Public Procurement), en la que se propone la metodología CCV como una herramienta básica de análisis. Reforzando estas posturas, un estudio sobre la contratación sostenible realizado en 2007 por el Öko-Institut por encargo de la propia Comisión Europea (40) se concluye que la adqui-

\footnotetext{
${ }_{3}$ Datos correspondientes a 2010, según informe publicado por Knight Frank. La media europea se ve rebasada en casos como el de Alemania, donde el porcentaje de vivienda en alquiler asciende al $57 \%$.
} 
sición de productos y servicios sostenibles no es necesariamente más onerosa para las administraciones públicas contratantes que la de productos no sostenibles, siempre que el proceso de contratación se lleve a cabo mediante la aplicación de análisis CCV.

A su vez, en el último informe sobre sistemas de contratación pública del Instituto Internacional para un Desarrollo Sostenible -IISD- (41) se constata que España no forma parte de los países que desarrollan políticas públicas para la implantación de dichos sistemas. En este documento se afirma que «los Gobiernos están descubriendo la necesidad de integrar el análisis del coste del ciclo de vida en las políticas públicas de contratación sostenible», así como que "el coste del ciclo de vida y el valor actual neto son particularmente importantes (en las políticas de contratación del sector público), ya que permitirán demostrar que, si bien los bienes y servicios social y medioambientalmente preferibles pueden tener unos costes de inversión iniciales superiores, se convertirán en la opción más rentable si se lleva a cabo un análisis del ciclo de vida integral de los proyectos, suponiendo ahorros sustanciales tanto durante la vida útil del inmueble como en el proceso de eliminación cuando esta llega a su final». Este es precisamente el problema que subyace en la aplicación de los análisis CCV en España, donde las consecuencias inmediatas de las perspectivas de importantes inversiones a corto plazo dominan, por los motivos que se han señalado, sobre las economías que se obtienen en una visión global durante toda la vida útil del inmueble.

\section{CONCLUSIONES}

La evaluación económica de los proyectos inmobiliarios más allá de la fase de construcción se convierte en una herramienta esencial para la optimización de costes, permitiendo la com- paración realista entre diferentes opciones de inversión: habrá multitud de niveles óptimos de rentabilidad dependiendo de cada edificio y de la propia perspectiva y expectativas del inversor sobre las condiciones de inversión aceptables.

La triple consideración del concepto de sostenibilidad -ambiental, social y económica-, y la generalización irreversible de este concepto ha definido la evolución de las metodologías de análisis financiero, incorporando la consideración del entorno en las evaluaciones de rentabilidad: en esta línea ha evolucionado la más reciente normativa europea de aplicación del análisis del Coste del Ciclo de Vida (CCV).

En el ámbito español, la Disposición final sexta del Real Decreto Legislativo 3/2011, de 14 de noviembre, por el que se aprueba el texto refundido de la Ley de Contratos del Sector Público, habilita al Gobierno para su desarrollo reglamentario, y en nada se opondría a las previsiones de la Ley la implantación de los análisis CCV en los sistemas de adjudicación, en concreto en lo relativo a la aplicación de su artículo 134.1. Esta podría ser, por lo tanto, una inmejorable oportunidad para introducir el método CCV en las políticas públicas de contratación y gestión patrimonial cumpliendo así las directrices de la Unión Europea, lo que pondría a España en la primera división de los países con sistemas públicos de contratación sostenible: de esta forma se impulsaría la utilización del método en el sector privado. Una iniciativa como esta requiere la adaptación el sistema a las características propias del sector de la construcción, y de la edificación en particular, para lo que habrá que preparar una norma española específica, construir un modelo propio con la consiguiente creación de bases de datos o sistematización de las que hoy están disponibles y desarrollar las herramientas informáticas precisas o adaptar las existentes en otras latitudes. Esta es la tarea que queda por delante.

\section{REFERENCIAS}

(1) Lützkendorf, T. (2010). Sustainable properties - dream or trend?. Informes de la Construcción, 62(517): 5-22, doi: http://dx.doi.org/10.3989/ic.08.055.

(2) WCED. (1987). Our common future. Report of the World Commission on Environment and Development. UK: Oxford University Press.

(3) Gluch, P., Baumann, H. (2004). The life cycle costing (LCC) approach: a conceptual discussion of its usefulness for environmental decision-making. Building and Environment, 39(5): 571-580, doi: http://dx.doi.org/10.1016/j.buildenv.2003.10.008.

(4) Klöpffer, W., Ciroth, A. (2011). Is LCC relevant in a sustainability assessment?. International Journal of Life Cycle Assessment, 16(2): 99-101, doi: http://dx.doi.org/10.1007/s11367-011-0249-y.

(5) Jørgensen, A., Hermann, I. T., Birk-Mortensen, J. (2010). Is LCC relevant in a sustainability assessment? Int J Life Cycle Assess, 15(6):531-532, doi: http://dx.doi.org/10.1007/s11367-010-0185-2.

(6) SMART SPP. (junio, 2011). Innovation through sustainable procurement. Tool for calculating Life-Cycle Costs and CO2 Emissions of products. http://www.smart-spp.eu/index.php?id=7633.

(7) CILECCTA - Construction Industry LifE Cycle CosT Analysis. http://www.cileccta.eu/.

(8) Departament of Industry-Comitte for Terotechnology. (1977). Life cycle costing in the management of assets : a practical guide. London: H.M. Stationery Office.

(9) Ruegg, R., McConnaughey, J. S., Thomas-Sav, G., Hockenbery, K. A. (1978). Life-Cycle Costing: A guide for selecting energy conservation projects for public buildings. Building Science Series, 113. Washington, D.C: National Bureau of Standards.

(10) Fuller, S.K., Petersen, S.R. (1995). Life-Cycle Costing Manual for the Federal Energy Management Program. Washington, DC: U.S. Department of Commerce, Technology Administration, National Institute of Standards and Technology.

(11) Rushing, A. S., Kneifel, J. D., Lippiatt, B. C. (2010, mayo). Handbook 135 and NBS Special Publication 709. Nacional Institute of Standards and Technology.

(12) Education Support Services/Facilities. (1999). Life Cycle Cost Analysis Handbook (1st Edition). State of Alaska: Department of Education \& Early Development. http://www.eed.state.ak.us/facilities/publications/LCCAHandbook1999.pdf.

(13) Australian National Audit Office. (2001, diciembre). Life Cycle Costing. Better Practice Guide. http://www.anao.gov. au/uploads/documents/Life_Cycle_Costing.pdf. 
(14) Kirkham, R., Alisa, M., Pimenta-da Silva, A., Grindley, T., Brondsted, J. (2004, septiembre). EUROLIFEFORM: An integrated probabilistic whole life cycle cost and performance model for buildings and civil infrastructure. RICS Foundation, COBRA 2004.

(15) Task Group 4. (2003, julio). Life-Cycle Cost in Construction. Final Report. UE: 3rd Tripartite Meeting Group on the Competitiveness of the Construction Industry. http://www.gci-uicp.eu/Documents/Reports/LCC\%20FINAL\%20REPORT-2.pdf.

(16) Davis Langdon Management Consulting. (2007, mayo). Life cycle costing (LCC) as a contribution to sustainable construction: a common methodology. Final Report. http://ec.europa.eu/enterprise/sectors/construction/competitiveness/life-cycle-costing/index_en.htm.

(17) Norwegian Standard Classification System. (2013). NS 3454:2013. Livssykluskostnader for byggverk - Prinsipper og struktur. Noruega.

(18) ISO. (2008). ISO 15686-5:2008(E) Buildings and constructed assets - Service-life planning - Part 5: Life-cycle costing. International Organization for Standardization.

(19) BCIS .(2008). Standardized Method of Life Cycle Costing for Construction Procurement. A supplement to ISO 156865:2008 Buildings and constructed assets - Service life planning - Part 5: Life cycle costing. Building Cost Information Service.

(20) InPro Building (Open Information Environment for collaborative Processes throughout the lifecycle of a building). Report - The InPro Lifecycle Design Framework for Buildings. http://www.inpro-project.eu/main.asp

(21) Davis Langdon. (2010, 19 de enero). Development of a Promotional campaign for Life-Cycle Costing in Construction. Final Report. http://ec.europa.eu/enterprise/sectors/construction/files/compet/life_cycle_costing/100119_development_of_a_promotional_campaign_en.pdf.

(22) UNEP. (2011). Towards a Lyfe Cycle Sustainability Assessment. United Nations Environment Program.

(23) CEN-AFNOR. CEN/TC 350 Sustainability of construction works. EN 15643. Sustainability assessment of buildings.

(24) AEN/CTN 198:9. (2011). UNE-EN 15643-1:2011 Sostenibilidad en la construcción. Evaluación de la sostenibilidad de los edificios.

(25) González-Méndez, J.J., Robles-Urquijo, I. (2010, marzo). CILECCTA. Herramientas de análisis de ciclo de vida, costes y opciones. En SB1omad Sustainable Building Conference. Congreso Internacional sobre Edificación sostenible. Madrid.

(26) Fawcett, W., Hughes, M., Hannes, K., Albrcht, S., Vennström, A. (2012). Flexible strategies for long-term sustainability under uncertaint. Building Research \& Information, 40(6): 545-557, doi: http:dx.doi.org/10.1080/09613218.2012.702 565.

(27) CEN. (2007). CEN/TC 228 - Heating systems in buildings. EN 15459:20o7. Energy performance of buildings - Economic evaluation procedure for energy systems in buildings.

(28) Hovde, P.J. (2005, 17-20 de abril). The Factor Method - A simple tool to service life estimation. En 1oDBMC International Conference On Durability of Building Materials an Component. Lyon. http://www.irbdirekt.de/daten/iconda/06059020143.pdf.

(29) UBS AG. Average useful life of building sections. Zurich. www.ubs.com/hypo.

(30) Arizona School Facilities Board, Arizona Gov. Average life Cycle of Building Components. http://www.azsfb.gov/sfb/ preventive\%20maintenance/life\%20expectancies.xls.

(31) University of Florida. (2010). Life Cycle Cost for Materials and Building Systems for Florida's Public Educational Facilities. http://www.fldoe.org/edfacil/pdf/lccgmbsfpef.pdf.

(32) Hong, T., Kim, J., Koo, C. (2012). LCC and LCCO2 analysis of green roofs in elementary schools with energy saving measures. Energy and Buildings, 45: 229-239, doi: http://dx.doi.org/10.1016/j.enbuild.2011.11.006.

(33) Chong, W.T., Naghavi, M. S., Poh, S. C., Mahlia, T. M. I., Pan, K. C. (2011). Techno-economic analysis of a wind-solar hybrid renewable energy system with rainwater collection feature for urban high-rise application. Applied Energy, 88(11): 4067-4077, doi: http://dx.doi.org/10.1016/j.apenergy.2011.04.042.

(34) Marszal, A.J., Heiselberg, P. (2011). Life cycle cost analysis of a multi-storey residential Net Zero Energy Building in Denmark. Energy, 36(9): 5600-5609, doi: http://dx.doi.org/10.1016/j.energy.2011.07.010.

(35) Uygunoglu, T., Kecebas, A. (2011). LCC analysis for energy-saving in residential buildings with different types of construction masonry blocks. Energy and Buildings, 43(9): 2077-2085, doi: http://dx.doi.org/10.1016/j.enbuild.2011.04.011.

(36) Schade, J. (2007). Life cycle cost calculation models for buildings. InPro (Open Information Environment for Knowledge-Based Collaborative Processes throughout the Lifecycle of a Building). http://www.inpro-project.eu/media/lcc_ juttaschade.pdf.

(37) Davis Langdon Management Consulting. (2007, mayo). Life cycle costing (LCC) as a contribution to sustainable construction: a common methodology, (p. 82). http://ec.europa.eu/enterprise/sectors/construction/competitiveness/lifecycle-costing/index_en.htm.

(38) European Commission. (2004). Buying Green! A handbook on environmental public procurement. http://ec.europa. eu/environment/gpp/pdf/buying_green_handbook_en.pdf.

(39) Commission of the European Communities. (2008). http://eur-lex.europa.eu/LexUriServ/LexUriServ. do?uri=COM:2008:0400:FIN:EN:PDF

(40) Öko-Institut e.V. (2007). Costs and benefits of Green Procurement in Europe. Freiburg. http://ec.europa.eu/environment/gpp/pdf/eu_recommendations.pdf.

(41) IISD. (2010, septiembre). Procuring Green in the Public Sector: A check-list pfor getting started. International Institute for Sustainable Development. http://www.iisd.org/pdf/2011/procuring_green_public_sector.pdf. 